\title{
DeCLARAción de Viena SOBRE LA INVESTIGACIÓN ARTíSTICA*
}

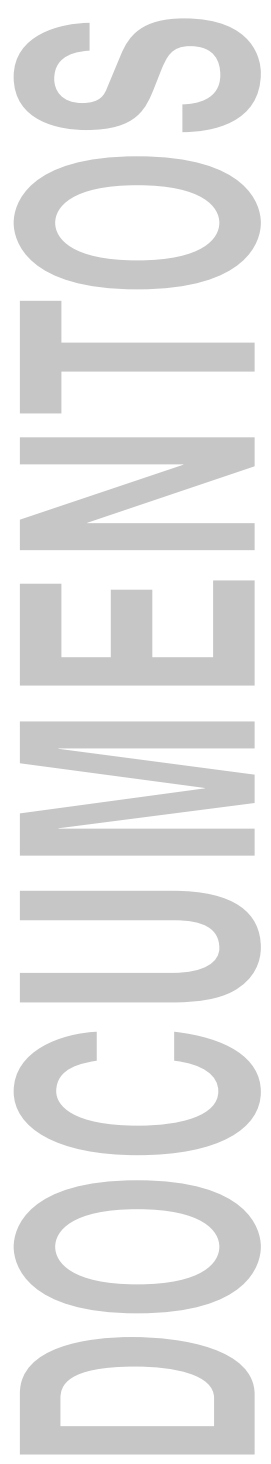

\section{INTRODUCCIÓN}

La investigación artística es un campo de investigación en las artes basado en la práctica y regido por esta, que ha experimentado un rápido desarrollo en todo el mundo en los últimos veinte años y que conforma una base de conocimientos clave para la formación artística en instituciones de educación superior en arte. La Declaración de Viena está concebida como un documento de principios dirigido a responsables políticos, organismos de financiación e instituciones de educación superior e investigación, así como a otras organizaciones e individuos que impulsen y realicen proyectos de investigación artística.

La declaración tiene como objetivo (1) articular de una forma más clara y acertada los conceptos y la repercusión de la investigación artística tal como se plantean en el Manual de Frascati -el manual de clasificación de la OCDE para recoger datos estadísticos y de investigación-. Esta aclaración garantizará que se realicen y reconozcan actividades de investigación exitosas en esta disciplina y, en consecuencia, contribuirá a (2) reestructurar las políticas y los programas de financiación en los ámbitos regional, nacional, europeo y mundial, de tal modo que apoyen la investigación artística en consonancia con las ciencias y las humanidades; y a (3) garantizar e incorporar en la educación superior en arte, en todos los países de Europa, estudios de tercer ciclo basados en la práctica, con el fin de seguir desarrollando la investigación artística y de afianzar el carácter contemporáneo del plan de estudios.

Los firmantes de la Declaración de Viena representan a los principales agentes actuales del ámbito de la investigación artística en Europa: las mayores organizaciones de instituciones de educación superior en arte representativas de disciplinas, la organización disciplinaria más importante que representa las principales iniciativas

*'Traducción del siguiente texto original: The Vienna Declaration on Artistic Research (2020). Texto co-escrito por AEC, CILECT / GEECT, Culture Action Europe, Cumulus, EAAE, ELIA, EPARM, EQ-Arts, MusiQuE, SAR, acceso 20 de noviembre de 2020, https://www.aec-music.eu/ about-aec/news/vienna-declaration-on-artistic-research. Traducción realizada por el Servicio de Traducción de la Universidad de Alcalá. 
en investigación artística, los dos organismos internacionales de garantía de la calidad de disciplinas artísticas y organizaciones políticas internacionales.

Hoy en día, el número de programas de doctorado dedicados a la investigación artística crece rápidamente por toda Europa, un crecimiento que viene respaldado por un creciente número de revistas académicas arbitradas sobre la disciplina en diferentes países; por un creciente número de proyectos ERASMUS + y Horizonte 2020 sobre este ámbito; y por una gran cantidad de publicaciones académicas que divulgan la investigación artística por todo el mundo.

\section{Principales Características de la inVestigación artística}

La excelencia en la investigación artística se alcanza por medio de la reflexión y la práctica artística de alto nivel; se trata de un estudio epistémico orientado a aumentar el conocimiento, el discernimiento, la comprensión y las destrezas. En este marco, la investigación artística se ajusta en todos los aspectos a los cinco criterios principales que constituyen la investigación y el desarrollo de acuerdo con el Manual de Frascati. A través de temas y problemas que tienen origen en la práctica artística y guardan relación con esta, la investigación artística también aborda cuestiones fundamentales de una trascendencia cultural, social y económica más amplia.

La investigación artística se lleva a cabo en todas las disciplinas de la práctica artística -incluidas la arquitectura, el diseño, el cine, la fotografía, las bellas artes, el arte multimedia y digital, la música y las artes escénicas- y obtiene sus resultados tanto en dichas disciplinas como, con frecuencia, en un ámbito interdisciplinario, combinando métodos propios con otros de otras tradiciones de investigación.

\section{Contexto}

Las instituciones de educación superior en arte operan predominantemente en un contexto de investigación y tienen la responsabilidad de llevar a cabo investigación artística. También es habitual que estas instituciones interactúen con departamentos de investigación y desarrollo de empresas relacionadas y que contribuyan directamente a la creación de propiedad intelectual en los ámbitos del arte, el entretenimiento y los medios a través de la práctica de investigación. Por estos motivos, las instituciones de educación superior en arte, que a menudo tienen un mandato gubernamental, están obligadas a ofrecer programas de enseñanza y aprendizaje basados en los conocimientos más recientes.

La repercusión de la investigación artística trasciende el sector de la educación superior y enlaza con diversos campos y comunidades profesionales, en particular, con los sectores cultural y creativo, 
así como con el educativo y el social. La investigación artística resulta idónea para estimular avances creativos e innovadores en sectores como la salud y el bienestar, el medio ambiente y la tecnología, por lo que contribuye a cumplir la «tercera misión» de las instituciones de educación superior en arte. Es preciso considerar el potencial único de la investigación artística en el desarrollo del «triángulo del conocimiento»-educación, investigación e innovación-, con el fin de aumentar la contribución de las instituciones de educación superior e investigación pública a la innovación, el compromiso social y el crecimiento económico. Históricamente, la investigación artística ha centrado su atención en la repercusión de su trabajo en diversos contextos fuera del ámbito académico, ya sea en la sociedad, la cultura, la economía o el medio ambiente.

\section{INFRAESTRUCTURA Y ACCESO A FINANCIACIÓN}

Dado que la investigación artística sigue siendo un campo relativamente reciente, en varios países está todavía por definir un marco de apoyo y financiación. En consecuencia, la investigación artística no goza en general del mismo acceso a la financiación que otros campos de investigación, o bien no puede optar a solicitar becas de investigación ni ayudas. No obstante, existen excepciones que podrían servir de modelo para establecer infraestructuras y culturas de investigación comparables a escala internacional por toda Europa y en otros continentes. Estos canales de financiación deberían incluir el apoyo al desarrollo continuo de la infraestructura de investigación, por ejemplo, formación de supervisores, resultados de investigación individuales basados en proyectos, procesos de garantía de la calidad y la creación de repositorios permanentes en los que al público en general le resulte más fácil descubrir trabajos de investigación y acceder a ellos, con el fin de alcanzar la sostenibilidad necesaria.

Las instituciones de educación superior en arte cada vez sienten una mayor motivación para realizar investigación artística; en este sentido, el desarrollo del entorno de investigación resulta esencial en un sector en maduración. Este objetivo tiene exactamente la misma importancia que los resultados de investigación y su repercusión, lo cual se ha convertido en una prioridad desde el punto de vista estratégico. Este entorno requiere financiación para formar a la próxima generación de investigadores mediante programas de doctorado, garantizar infraestructuras físicas y virtuales adecuadas, así como medios de archivado y divulgación, y establecer vínculos con negocios y grandes empresas para potenciar la repercusión de la investigación.

La investigación artística incorpora numerosos aspectos y rasgos que no están en formato textual, o no exclusivamente, como objetos, movimientos y sonidos. Los investigadores necesitan varias plataformas de presentación que combinen estos aspectos y rasgos de forma apropiada y, por consiguiente, se alejen del formato estándar de los artículos de publicaciones o los repositorios o archivos de investigación, o bien lo amplíen. 


\section{EVALUACIÓN Y RECONOCIMIENTO}

La investigación artística se valida mediante una evaluación externa realizada por especialistas del campo de competencias disciplinarias propio de cada trabajo. La garantía de la calidad es responsabilidad de organismos internacionales independientes y reconocidos y asegura los estándares que se describen en el documento Criterios y directrices para el aseguramiento de la calidad en el Espacio Europeo de Educación Superior (ESG). Los programas de doctorado existentes en investigación artística siguen los estándares establecidos que se describen en los Principios de Florencia de 2016, los cuales, a su vez, se basan en los documentos de política de educación doctoral del Proceso de Bolonia (por ejemplo, los Sal々̧burg Principles [Principios de Salzburgo] de 2005 y 2010, publicados por la EUA).

\section{LLAMAMIENTO A LA ACCIÓN}

Las entidades firmantes del presente documento piden que todas las partes implicadas adopten las siguientes medidas:

- Apoyar y fomentar la consolidación de la investigación artística como categoría independiente en el Manual de Frascati, brindando la oportunidad de recoger datos y estadísticas de investigación en este ámbito.

- Garantizar que las políticas y los programas de financiación, tanto nacionales como internacionales, incluyan a la investigación artística, proporcionen la infraestructura y los recursos necesarios y tengan presente la existencia de conocimientos en investigación artística en todos los paneles pertinentes de toma de decisiones.

- Garantizar que el abanico de resultados de investigación artística se reconozca plenamente a escala nacional e internacional y opte a procedimientos formales de garantía de la calidad o evaluación profesional.

- Garantizar, mediante la legislación pertinente, la creación de marcos jurídicos que permitan a las instituciones de educación superior en arte ofrecer programas de estudio de tercer ciclo y títulos apropiados en investigación artística.

AEC, CILECT / GEECT, Culture Action Europe, Cumulus, EAAE, ELIA, EPARM, EQ-Arts, MusiQuE, SAR 